Objectives This is a observational study with the aim to evaluate predictive factors of response to NAT in patients with breast cancer.

Methods It is a retrospective study included 21 patients $t$ who received neaoadjuvant chemotherapy between 2015 and 2019 at salah azaiez institute Tunisia., we collected, socociodemographics (age, gender, and marital status); tumor: localization, staging.

Results Our study included 21 patients who achieved breast pCR, between 2015 and 2019, 21 patients were included. The median age was 48 years (ranging 30-68). All patients had breast cancer and received neoadjuvant chemotherapy. Two patients were diabetic, 3 patients had high blood pressure and 2 had dyslipidemia. The majority of the tumor had a hign grad nuclear (14). The majority of molecular profile was tripe negative (6cases). The evaluation of the response based on imaging firstly and histological examination. Clinically all patient had a complete response. 17 patients underwent radical surgery and 4 conservative surgery. The evaluation of these patients showed that 16 of theme developed recurrence. We concluded that age, nuclear grad, histological type did not effected the response of chemotherapy but this results is insufficient because of the shortage of the serie.

Conclusions To date, no tumour biological factor is available for clinical use in the prediction of chemotherapy response in advanced breast cancer other than oestrogen receptor status, which predicts response to hormonal therapy

\section{EPV030/\#572 NUTRITIONAL STATUS AND QUALITY OF LIFE OF BREAST CANCER PATIENTS NEEDING FOR RESPONSE TO NEOADJUVANT CHEMOTHERAPY: ABOUT 19 PATIENTS}

M Bouhani*, S Kammoun, S Sakhri, H Bouaziz, I Bettaieb, A Goucha, R Chargui, M Driss. Salah Azaiez Institute of Oncology, Surgical Oncology, Tunis, Tunisia

\subsection{6/ijgc-2021-IGCS.97}

Objectives The aims of this study were to report nutritional status in 19 patients with cancer requiring neoadjuvant chemotherapy and to study the influence of nutritional status on their quality of life.

Methods It is a retrospective study included 19 patients $\mathrm{t}$ who received neaoadjuvant chemotherapy between 2015 and 2019 at salah azaiez institute Tunisia., we collected the nutritional status (weight, anorexia grading, type of diet),socociodemographics (age, gender, and marital status); tumor: localization, staging; health status (performance status according to WHO classification, usual weight and body mass index (BMI).

Results Between 2015 and 2019, 19 patients were included. The median age was 52 years (ranging 30-72). All patients had breast cancer and received neoadjuvant chemotherapy. 13 patients were menopaused, two patients were diabetic, 14 patients had high blood pressure and 4 had dyslipidemia. BMI was normal in 6 cases, overweight in 4 cases and obesity in 9 cases. The majority of the tumor were classified T4B. 17 patients had invasive ductal carcinoma. During chemotherapy, 3 patients follow vegetables and fruit diets, 7 follow mixed diets and 9 follow western diet.Three patient had a sport activity, sedentary lifestyle was noted in 9 patients et 7 patients had a normal daily activity. The evaluation of the response based on imaging firstly and histological examination.

Conclusions The nutritional status of patients with cancer requiring neaoadjuvant chemotherapy was relatively preserved. Functional impairment, the presence of anorexia, appear to be independent predictive factors of quality of life in patients who will recivied neaoadjuvant chemotherapy.

\section{EPV031/\#576 PAGET'S DISEASE OF THE NIPPLE: WITH CONCOMITANT BREAST TUMOR: ABOUT 10 CASES}

M Bouhani*, S Sakhri, S Kammoun, H Bouaziz, A Goucha, R Chargui, M Driss. Salah Azaiez Institute of Oncology, Surgical Oncology, Tunis, Tunisia

\subsection{6/ijgc-2021-IGCS.98}

Objectives Paget's disease is an uncommon breast malignancy and often misdiagnosed, it is associated with underlying in situ or invasive breast cancer. The objective of this study is to identify the type of underlying cancer and specify these characteristics.

Methods Nine patients with Paget's disease who were admitted to our hospital were analyzed retrospectively.

Results Our study included nine patients. Six patients were menopaused. Only seven patients presented with clinical findings suggestive of Paget's disease of the breast. The mean size of the tumor was $36 \mathrm{~mm}$ and axillary lymph node were found in seven cases and Mammography and ultrasonography were was performed in all 16 patients and ultrasonography,of the nine mammographic studies, three were negative, in the others cases it showed suspected opacity in three cases, pleomorphic microcalcifications in four cases and both opacity and microcalcifications in three cases.Four patients had multifocality or multicentricity. Modified radical mastectomy was performed in seven patients, mastectomy and sentinel lymph node dissection in two cases in two, and wide local excision with lymph node dissection in one patient. Pathological findings were ductal carcinoma in situ (DCIS) $(n=4)$, invasive ductal carcinoma (IDC) $(\mathrm{n}=1)$, invasive lobular carcinoma $(\mathrm{n}=1)$, DCIS with IDC $(\mathrm{n}=4)$. All tumors had a high nuclear grad.

Conclusions Patients with Paget's disease of the breast have a high incidence of an underlying breast carcinoma. Most of the patients in this study presented late and were more likely to have positive mammograms.

\section{EPV032/\#579 4 BI-RADS MICROCALCIFICATIONS OF THE BREAST: HOW DOES RADIOLOGIC CLASSIFICATION CORRELATE WITH HISTOLOGY?}

M Bouhani*, S Sakhri, F Abidi, S Kammoun, O Jaidane, R Chargui, K Rahal. Salah Azaiez Institute of Oncology, Surgical Oncology, Tunis, Tunisia

10.1136/ijgc-2021-IGCS.99 